Check for updates

Cite this: Phys. Chem. Chem. Phys., 2019, 21, 14832

Received 1st May 2019,

Accepted 13th June 2019

DOI: $10.1039 / c 9 c p 02474 c$

rsc.li/pccp

\section{Stable, one-dimensional suspended and supported monatomic chains of pnictogens: a metal-insulator framework $\dagger$}

\author{
F. Ersan, (D) ${ }^{a b}$ E. Aktürk (DD ${ }^{* a c}$ and S. Ciraci*b
}

Group-VA elements $\mathrm{P}, \mathrm{As}, \mathrm{Sb}$, and $\mathrm{Bi}$ can construct free-standing, stable zigzag monatomic chain structures, which show unusual properties. They are normally semimetals with bands crossing at the Fermi level, but a very narrow gap opens due to spin-orbit coupling. They attain one quantum of conductance under a small bias potential; Bi, being an exception, attains two quanta of conductance. Finite size chains are magnetic semiconductors; their magnetic moments and the order of spin states show an even-odd disparity depending on the number of chain atoms. Variations of the HOMO-LUMO band gaps depending on the spin polarization and the size of the finite chains offer critical tunability. In the periodic, zigzag compound chains, a small band gap opens at the Fermi level. The mysterious zigzag geometry, cohesion, stability and band order of all these chains are well-explained by a simple bond model. When placed on the parent or other monolayers like graphene, h-BN and GaSe, these chains become weakly bound and construct a 1D metallic channel. The artificial grids or networks of these metallic chains on the insulating substrates can constitute metal-insulator frameworks of desired geometry. The zigzag phosphorene chain, having the highest stability, remains stable even at full coverage of adsorbates like $\mathrm{H}$ and $\mathrm{OH}$, whereas other chains dissociate. While P-chains can be synthesized on GaSe and graphene substrates, phosphorene nanoribbons can transform into suspended chains under excessive tensile strain. Additionally, we showed that As, Sb, and Bi zigzag chains are weakly bound to their parent monolayers and remain stable. Nitrogen monatomic chains, on the other hand, are prone to instability. The diverse properties unveiled in this study based on the density functional method offer tunability through electric fields and strain.

\section{Introduction}

Dimensionality and size are important features of matter to control its physical properties. By going from the three-dimensional (3D) bulk to two-dimensional (2D) surfaces, then to slabs and finally to strictly 2D monolayer structures, the physics changes dramatically. Understanding the effects of dimensionality lowering from 3D to 2D has led to huge research with numerous fundamental discoveries. In the meantime, interest shifted to also reducing the dimensionality further from $2 \mathrm{D}$ to quasi one-dimensional (1D) like nanotubes, nanorods, and nanoribbons and also to strictly 1D atomic chains. While 3D diamond crystals are an insulator, 2D monolayers of carbon, i.e., graphene, behave as a massless Dirac fermion with

\footnotetext{
${ }^{a}$ Department of Physics, Adnan Menderes University, Aydn 09010, Turkey

${ }^{b}$ Department of Physics, Bilkent University, Ankara 06800, Turkey.

E-mail: ciraci@fen.bilkent.edu.tr

${ }^{c}$ Nanotechnology Application and Research Center, Adnan Menderes University, 09010 Aydn, Turkey. E-mail: ethem.akturk@adu.edu.tr; Fax: +90 2562135379; Tel: +902562130835

$\dagger$ Electronic supplementary information (ESI) available. See DOI: 10.1039/c9cp02474c
}

energy bands crossing linearly at the Fermi level. ${ }^{1}$ Even more dramatic is that strictly $1 \mathrm{D}$ carbon chains display extraordinary features; they become better conductors than gold chains. ${ }^{2-5}$ Observation of the quantization of conductance ${ }^{6}$ through a constriction like the contact between a scanning tunneling microscope (STM) tip and the metal surface ${ }^{7}$ and theories developed thereof ${ }^{8}$ made strictly 1D metallic chains an attractive subject of research. After the realization of stable gold monatomic chains suspended between two gold electrodes, ${ }^{9,10}$ the structure and the stability of diverse monatomic wires were investigated theoretically. ${ }^{11-14}$

While research on metal monatomic chains is progressing, the synthesis of carbon nanotubes ${ }^{15}$ and then graphene ${ }^{1}$ created a renewed interest not only in carbon monatomic chains, ${ }^{16-19}$ but also in other group-IV element and III-V compound chains. ${ }^{20}$ Further to the stability issues and quantized conductance, half-metallic behaviors and also size dependent ferromagneticantiferromagnetic alternation were reported for monatomic chains of carbon-transition metal compounds. ${ }^{16,21}$ In the meantime, research shifted to magnetization, not only in carbon chains, ${ }^{22}$ 
but also in chains of transition metal atoms. ${ }^{23}$ Phononic energy transfer and possible quantization of heat transfer through atomic chains were also other subjects of interest. ${ }^{24-27}$ A recent theoretical study predicted that zigzag Bi chains can occur on the GaAs(110) surface and this results in giant spin splitting with the in-plane and out-of-plane spin components. ${ }^{28}$

$1 \mathrm{D}$ atomic chains can grow on semiconducting surfaces through several different methods, these are: STM lithography, ${ }^{29}$ evaporation, ${ }^{30}$ epitaxial growth of metal rows, ${ }^{31}$ and solution methods. ${ }^{32}$ Recently, 1D phosphorus atomic chains were obtained experimentally from black phosphorene via electron beam sculpturing inside a transmission electron microscope. ${ }^{33}$ At the same time, $\mathrm{Xu}$ et al. reported that an ordered 1D structure of adsorbed phosphorus atoms can grow at low coverage on $\mathrm{Au}(111)$ through the molecular-beam epitaxy method. ${ }^{34}$ The formation of a well-defined zigzag chain is hindered by the strong interaction between $\mathrm{P}$ adatoms and $\mathrm{Au}$ atoms of the substrate. Bismuth dimers in the nanoline are observed experimentally on the $\mathrm{Si}(001)$ surface, ${ }^{35}$ or on $\mathrm{H}$ passivated $\mathrm{Si}(100)$ surfaces. ${ }^{36}$

Very little is known about the monatomic chains of pnictogens $(\mathrm{N}, \mathrm{P}, \mathrm{As}, \mathrm{Sb}, \mathrm{Bi})^{34,37,38}$ despite their $2 \mathrm{D}$ monolayers, multilayers and nanoribbons having received growing interest. ${ }^{39-51}$ In fact, $2 \mathrm{D}$ mono- and multilayers of pnictogens offer diverse properties to be used in various applications, such as in topological spintronic devices, biosensors, batteries, thermoelectric devices, nonlinear optics etc. For example, $\mathrm{P}$ and As monolayers and multilayers are semiconductors and hence become attractive materials in $2 \mathrm{D}$ electronics. ${ }^{40}$ On the other hand, monolayers of $\mathrm{Sb}$ and $\mathrm{Bi}$ and their binary compounds with significant spin-orbit coupling exhibit topologically non-trivial behaviors, ${ }^{52,53}$ which are of fundamental importance. It is therefore interesting to know how the properties of $2 \mathrm{D}$ pnictogen monolayers change when the dimensionality is reduced from two to one. Additionally, pnictogen chains formed on diverse substrates, in particular on host monolayers, may form composite structures and 2D frameworks with interesting features.

Our study is based on density functional theory (DFT) and comprises periodic and finite size monatomic chains of $\mathrm{N}, \mathrm{P}$, $\mathrm{As}, \mathrm{Sb}$, and $\mathrm{Bi}$, periodic chains of their binary compounds like $\mathrm{NP}$, PAs, AsSb, and SbBi and periodic chains on diverse substrates together with the simulation of their synthesis. We provide accurate answers to the following crucial questions, which have been frequently raised: what are the stable structures of free-standing chains? Can their properties and stability be maintained when they occur on selected substrates? Can the free-standing and supported atomic chains be synthesized? The important and new findings of our work are summarized as follows: (i) the zigzag geometry is the lowest energy configuration for the monatomic chains of $\mathrm{P}, \mathrm{As}, \mathrm{Sb}$ and $\mathrm{Bi}$. The bonding of this mysterious geometry is unveiled by a simple bond orbital model constructing two $\sigma$ - and one $\pi$-bond between two pnictogen atoms. Strong cohesion, the ordering of the bands, in particular those near the Fermi level, and their folding with the opening of a very narrow gap at the zone boundary are explained by this simple bond orbital model. (ii) The order of spin states, corresponding band gaps, and the total magnetic moments of finite size zigzag chains show interesting even-odd disparity of the number of atoms in the chain. (iii) The compounds of group-VA elements form also periodic, stable zigzag chains; they are narrow band gap semiconductors. (iv) Zigzag chains are weakly bound to their parent monolayers and monolayers like graphene, h-BN and GaSe, but they are thermally stable. These chains open 1D metallic channels or interconnect on the insulating substrates, and also allow the construction of metal-insulator frameworks of desired geometries. (v) P atoms which are randomly placed on graphene substrates can reconstruct to form a zigzag chain of $\mathrm{P}$. The freestanding $\mathrm{P}$ chain can also form under excessive tensile strain of the parent phosphorene nanoribbons. (vi) While free-standing zigzag monatomic chains are dissociated when foreign adsorbates like $\mathrm{H}$ and $\mathrm{OH}$ are adsorbed onto each chain atom, the zigzag phosphorus chain, as an exception, remains stable, but its electronic structure is modified. (vii) Nitrogen chains behave differently from those of the rest of the group-VA elements.

We believe that zigzag metallic pnictogen chains weakly bound to specific insulating substrates can construct $1 \mathrm{D} / 2 \mathrm{D}$ composite structures (heterostructures) or metal-insulator frameworks of desired geometries offering crucial conduction properties. With the fundamental background acquired through the present theoretical predictions we aim to set a platform for new experimental studies.

\section{Computational details}

Our predictions have been obtained from theoretical analysis based on first-principles plane-wave calculations within density functional theory for both spin-polarized and spin-unpolarized cases. All numerical calculations are performed with the Vienna $a b$ initio simulation package (VASP) software. ${ }^{54}$ In this study, we used the projector-augmented wave (PAW) potentials ${ }^{55}$ and the exchange-correlation potential is approximated with the PerdewBurke-Ernzerhof (PBE)-functional, ${ }^{56}$ including the D2-Grimme type van der Waals (vdW) correction. ${ }^{57}$ A plane-wave basis set with a kinetic energy cutoff of $400 \mathrm{eV}$ is used in the calculations, except systems including oxygen atoms where the cutoff energy is increased to $600 \mathrm{eV}$. The Brillouin Zone (BZ) of the structures was sampled in $\mathbf{k}$-space within the Monkhorst-Pack scheme ${ }^{58}$ by a $(16 \times 1 \times 1)$ mesh. The vacuum spacing between successive atomic chains is kept larger than $16 \AA$. The energy convergence value between two successive steps was smaller than $10^{-5} \mathrm{eV}$, and a maximum Hellmann-Feynman force of $0.001 \mathrm{eV} \AA^{-1}$ was allowed on each atom. Spin-orbit coupling (SOC) is taken into account in all calculations, which becomes crucial already for arsenic atoms. The band gaps are corrected over the PBE results by using the Heyd-Scuseria-Ernzerhof (HSE) hybrid functional. ${ }^{59,60}$ The screening length of HSE is $0.2 \AA$, and the mixing rate of the HF exchange potential is 0.25 . Calculations resulting in a magnetic state are reproduced also by Siesta ${ }^{61}$ software, which uses a local basis set.

The character of the bonding in the chain structure is analyzed in terms of the total charge density, $\rho_{\mathrm{T}}$, and the charge density difference, $\Delta \rho$, which is obtained by subtracting the charge density of the constituent atoms from $\rho_{\mathrm{T}}$. To plot the charge distributions 0.02 e $\AA^{-3}$ isosurface levels are used. 
Further to the structure optimization, the analyses of dynamical stability are carried out by calculating the phonon frequencies in terms of the density functional perturbation theory method as implemented in the PHONOPY ${ }^{62}$ code with and without the SOC term. The stability of the optimized structures against thermal excitations is tested using finite-temperature $a b$ initio molecular dynamics (AIMD) calculations. A Nosé thermostat was used, and Newton's equations of motion were integrated through the Verlet algorithm with time steps of 1 fs. All structures considered in this study are treated in $21 \times 1 \times 1$ supercells and kept at $300 \mathrm{~K}$ and $500 \mathrm{~K}$ for $4 \mathrm{ps}$.

The cohesive energies of the stable structures are calculated using the expression $E_{\mathrm{c}}=\left[n E_{\mathrm{VA}}-E_{\text {chain }}\right] / n$, in terms of the total energy of the constituent free group-VA atom $E_{\mathrm{VA}}$ and the optimized total energy of the chain $E_{\text {chain. }} n$ is the number of atoms in the cell. $E_{\mathrm{c}}>0$ indicates cohesion of the given structure. The binding energy of $\mathrm{H}, \mathrm{O}$ and $\mathrm{OH}$ adsorbed onto a chain is calculated using the expression $E_{\mathrm{b}}=\left[E_{\mathrm{A}}+E_{\text {chain }}-E_{\text {chain }+\mathrm{A}}\right]$, in terms of the total energies of the free adatom (or molecule), bare chain and adsorbed adatom chain, respectively.

\section{Results}

\subsection{Structures and stability of monatomic chains}

In this study, we are considering six different types of structures for the suspended, monatomic, infinite (periodic) chains of group-VA elements ( $\mathrm{N}, \mathrm{P}, \mathrm{As}, \mathrm{Sb}$ and $\mathrm{Bi}$ ) as illustrated in Fig. 1(a). They are labeled as the linear chain (L), Peierls chain (Peierls), armchair (AC), armchair-zigzag mixed chain, (AC-ZZ), reconstructed zigzag (ZZ-R) and zigzag chain (ZZ). The cohesive energies of these six different types of chains calculated for each element are shown in Fig. 1(b). In addition to the chain structures, we also calculated the cohesive energy of the dimer of group-VA elements for the sake of comparison. It was found that ZZ-chains are most favorable energetically among the six different types of chain structures, except for $\mathrm{N}$ atoms, which preferably dimerise to form $\mathrm{N}_{2}$ molecules (which is the strongest bond ever). That the P-ZZ chain has the strongest cohesion is in compliance with recent synthesis of narrow nanoribbons, which were aligned exclusively in the zigzag crystallographic orientation, indicating the enhanced stability of the zigzag geometry. ${ }^{63}$ On the other hand, linear chains are least favorable energetically and are prone to instability. Generally, the heavier the constituent, the weaker is the cohesion.

Having determined the optimized structures for all six monatomic chains we analyze their stability. The dynamical stability at $T=0 \mathrm{~K}$ is tested by calculating the frequencies of the vibration modes in the Brillouin zone. The top panels of Fig. 2 show the phonon dispersions of the dynamically stable chains. These are AC-ZZ monatomic chains of $\mathrm{P}$ and As, and zigzag (ZZ) monatomic chains of $\mathrm{P}, \mathrm{As}, \mathrm{Sb}$ and $\mathrm{Bi}$. The cohesive energies of $\mathrm{P}$ - and As-AC-ZZ are slightly lower than the corresponding zigzag chains, P- and As-ZZ. The rest of the structures attain imaginary frequencies and are unstable at $T=0 \mathrm{~K}$. The overall $\Omega(k)$ dispersions of the phonons are similar, except that the
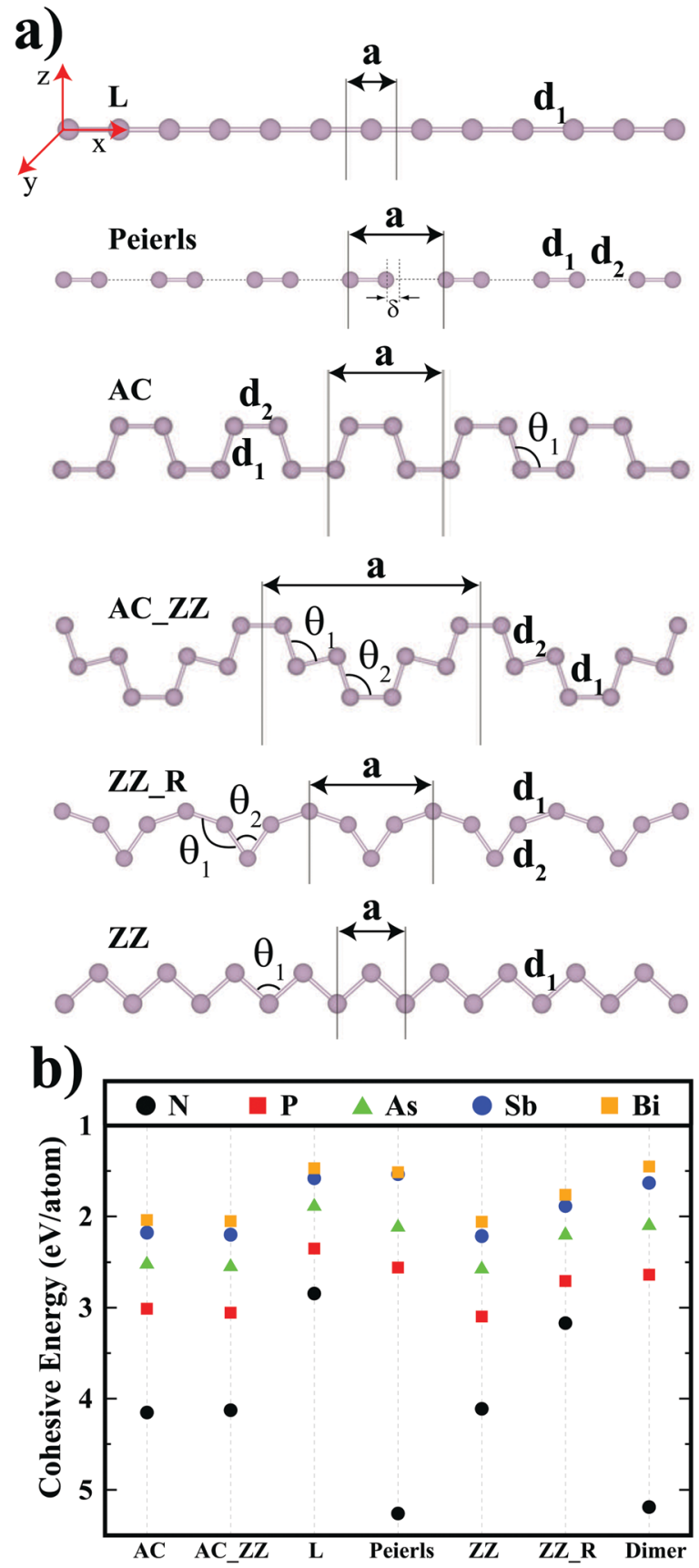

Fig. 1 (a) Side views of six different types of structures for suspended, monatomic, periodic (infinite) chains of group-VA elements, N, P, As, Sb, and $B i$ : the linear chain $(L)$, dimerised linear chain (Peierls), armchair chain (AC), armchair-zigzag mixed chain (AC-ZZ), reconstructed zigzag chain (ZZ-R) and zigzag chain (ZZ). 1D primitive unit cells of the chains are delineated. 1D lattice constants and relevant structural parameters of each type are also marked. (b) Optimized cohesive energies of the six different chain structures described in (a) and also those of the single dimers of group-VA elements.

frequencies of acoustic and optical modes vary depending on the strength of the bonds between nearest atoms. It should be noted that with increasing row numbers and atomic weights, the bond lengths become longer and hence the force constants get weaker. This leads to relatively lower vibration frequencies for the chains constituting heavier atoms. 

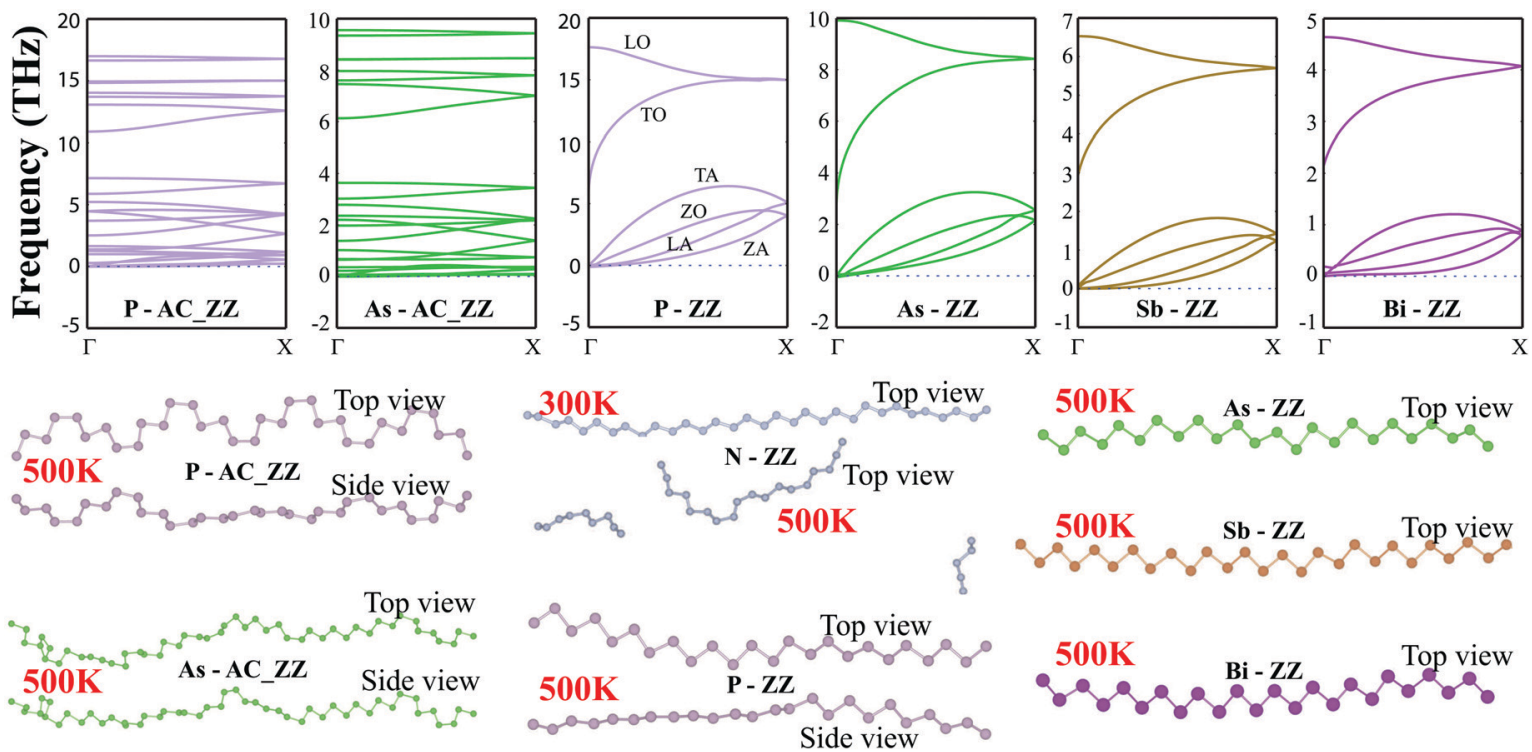

Fig. 2 Top: Phonon dispersions of the stable chains. Bottom: Snapshots of AIMD simulations of the dynamically stable chains after 4 ps. The N-ZZ chain is dissociated at $T=500 \mathrm{~K}$.

The stability of the chains against thermal excitations is also tested by AIMD calculations for all structures, even if some of them had imaginary frequencies. In compliance with the dynamical stability tests, dynamically unstable chain structures were also broken down into small pieces or clusters at the end of the AIMD simulations at $500 \mathrm{~K}$ for $4 \mathrm{ps}$. The bottom panels of Fig. 2 are the snapshots of the AIMD simulations. As seen, although the N-ZZ chain has imaginary phonon frequencies in the long wavelength limit, it can keep the zigzag pattern intact at a $300 \mathrm{~K}$ temperature (for $4 \mathrm{ps}$ ), but it breaks into small finite chains at $500 \mathrm{~K}$ for $4 \mathrm{ps}$. This situation can be explained by the relatively short simulation time, wherein $\mathrm{N}-\mathrm{ZZ}$ was not able to break down. While the P-AC-ZZ chain keeps its mixed pattern, the As-AC-ZZ chain transforms into the lower energy ZZ chain structure in the course of the AIMD simulations. Notably, all stable ZZ chain structures gain kinetic energy with increasing temperature and start to rotate around the $x$-axis, keeping their zigzag configuration. Calculated values related to their structural parameters and energetics are summarized in Table 1 for both dynamically and thermally stable chains. Despite its dynamical instability, N-ZZ is also included in the table for the sake of comparison. Also the cohesive energies of $2 \mathrm{D}$ monolayers of group-VA elements in buckled honeycomb, symmetric or asymmetric washboard and square-octagon structures are given for comparison. ${ }^{64-69}$ As expected, the cohesive energies of stable monatomic chains are slightly smaller than those of the corresponding monolayers, but they are rather high due to triple bonds between atoms as discussed in the next section. Also, the bond length of a 1D monatomic chain is shorter than that of the corresponding 2D monolayer. This explains the stability and high cohesive energy of free-standing, monatomic chains of group-VA atoms despite their lower coordination numbers relative to the parent monolayers, and indicates that their synthesis can be achieved.

The stiffness of the chains under uniaxial strain is also crucial. To this end we calculated the variation of the total energy of P-ZZ with the strain defined as $\varepsilon=100 \times\left(a_{i}-a_{0}\right) / a_{0}$. Fig. 3 presents the variation of the total energy of the P-ZZ chain

Table 1 Structural parameters and relevant energies calculated for the stable, monatomic group-VA chains: lattice constant (a); bond length in the 1D chain structure $\left(d_{\text {chain }}\right)$; bond length in the 2D monolayer; bond angle $(\theta)$; the cohesive energy in the chain structure $\left(E_{c}\right)$; the cohesive energy of the corresponding 2D monolayer in buckled honeycomb (hb), symmetric (sw) or asymmetric (aw) washboard and square-octagon (so) structures $\left(E_{\mathrm{c} ; 2 \mathrm{D}}\right)$; the band gap $\left(E_{\mathrm{g}}\right)$ (with $(d)$ indicating a direct band gap and M: metal, SM: semimetal); the band gaps corrected by HSE; and the elastic stiffness coefficient ( $\kappa$ )

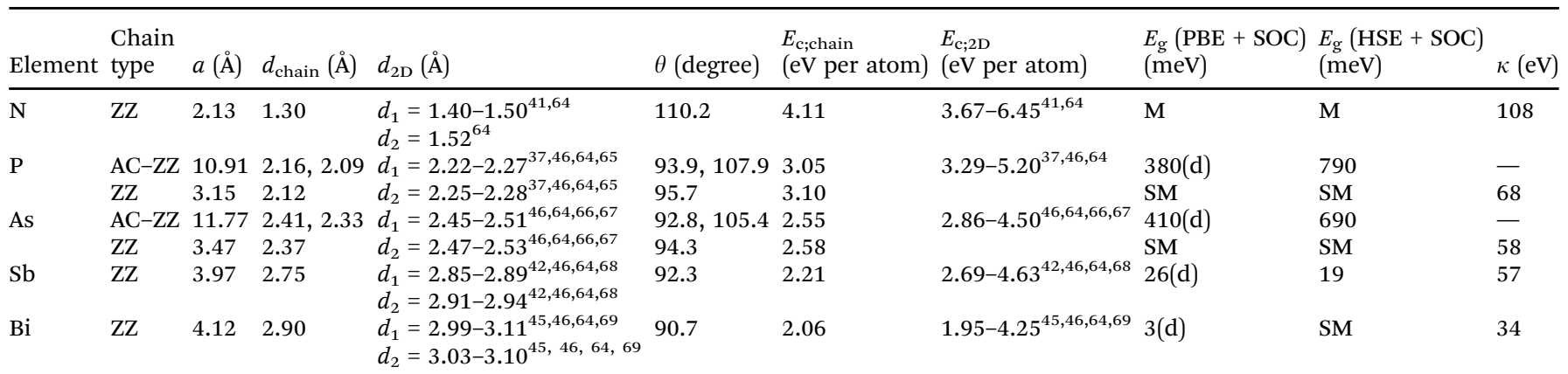




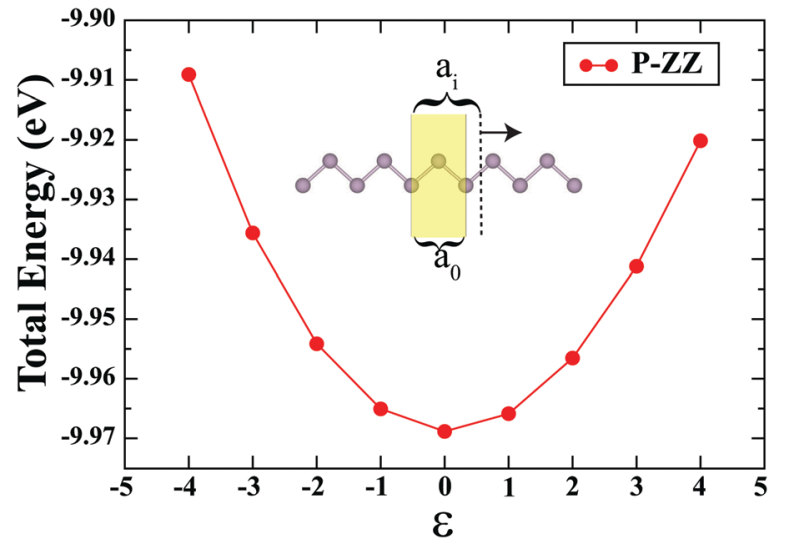

Fig. 3 Variation of the total energy per unit cell of the P-ZZ chain with compressive and tensile strain, $\varepsilon$.

with the axial strain. Here $a_{i}$ is the strained lattice constant. Other $\mathrm{ZZ}$ chains exhibit also similar parabolic curves for the variation of the total energy with strain, $\varepsilon$. The elastic stiffness of the $\mathrm{ZZ}$ type chains is then defined by the second derivative of the total energy per unit cell with respect to the axial strain $\left(\kappa=d^{2} E_{\mathrm{T}} / d^{2} \varepsilon\right)$. The calculated elastic stiffness decreases from $\kappa=108 \mathrm{eV}$ to $\kappa=34 \mathrm{eV}$ going from $\mathrm{N}$ to Bi. Notably, the average of the elastic stiffness values of group-VA $\mathrm{ZZ}$ chains is much smaller than that of carbon nanotubes. ${ }^{70}$

\subsection{Electronic structures of stable periodic chains}

While monatomic chains are normally prone to Peierls instability, the chain structure undergoing a Peierls deformation was found to be unstable for $\mathrm{P}, \mathrm{As}, \mathrm{Sb}$ and $\mathrm{Bi}$ in the present study. Instead ZZ- and AC-ZZ-chains were found to be stable. Here we will address two crucial questions of why the zigzag geometry is preferred and whether the chains having even numbers of valence electrons in a unit cell are semiconductors or metals. Finally, we will clarify the character of the bonds between chain atoms. Fig. 4(a) shows the band structure and total, as well as partial, densities of states (PDOS) of stable monatomic chains calculated within PBE + SOC. Again, the electronic structure of $\mathrm{N}-\mathrm{ZZ}$ is shown for the sake of comparison. All the bands of ZZchains look similar except those near the Fermi level. While the $\mathrm{N}-\mathrm{ZZ}$ chain is a metal, the P-ZZ, As-ZZ, Sb-ZZ and Bi-ZZ chains display a semimetallic property with band gaps ranging between $10 \mathrm{meV}$ and $20 \mathrm{meV}$. The band gaps of Sb-ZZ and Bi-ZZ are found to be $19 \mathrm{meV}$ and $3 \mathrm{meV}$, when they are calculated also by applying the correction using a hybrid functional (HSE) to the PBE band and by including SOC. Apparently, SOC has closed the band gap of the Bi-chain. Perfect and periodic zigzag chains of $\mathrm{P}, \mathrm{As}$, and $\mathrm{Sb}$ allow ballistic transport where the phases of the current carrying states are maintained and hence can attain one quantum of conductance under a small bias. Whereas, periodic Bi-ZZ can attain two quanta of conductance under a small bias. In Table 1 , the band gaps of $\mathrm{AC}-\mathrm{ZZ}$ and $\mathrm{ZZ}$ monoatomic chains of pnictogens calculated by PBE + SOC and HSE + SOC are presented.

A simple and schematic picture of bonding and band formation is described in Fig. 4(b) for the ideal bond angle, $\theta=90^{\circ}$.
The s-orbitals form bonding and antibonding states, from where the lowest two filled bands are derived. Neighboring $\mathrm{p}_{x}$ and $\mathrm{p}_{y}$ orbitals can form ideal and perpendicular bonds and hence the corresponding two bands above the s-bands. Two s-bands, as well as $\mathrm{p}_{x}, \mathrm{p}_{y}$-bands, are well separated at the center of the BZ, but they join at the $X$-point. Bands of ZZ-chains originating from bonding and antibonding $\pi$-bonds of $\mathrm{p}_{z}$-orbitals are again wide open at the $\Gamma$-point, but become very close at the $X$-point, except for Bi-ZZ; the maxima of its valence bands occur both at the $\Gamma$ - and $X$-points of the BZ. Deviations from the ideal angles give rise to overlap of adjacent bonds and hence metallization sets in. ${ }^{71}$ Owing to the relatively smaller bond distance of $\mathrm{N}-\mathrm{ZZ}, \mathrm{s}-$ and $\mathrm{p}$-bands are highly dispersed. The metallic state of the N-ZZ chain is an indication of instability. However, as the bond lengths $d_{\mathrm{v}-\mathrm{v}}$ increase with the row number, the dispersions of $\mathrm{p}_{x, y, z^{-}}$and s-bands decrease and a lower lying s-band constructed from antibonding s-orbitals becomes separated from the higher lying p-bands. Nonetheless, since the gap between the s-band and p-bands is small for P-ZZ, these bands, in particular the s-band and $\mathrm{p}_{x}$-band at the center of the BZ, show significant hybridization by exchanging their orbital character. At this point, the question of why the ZZ-structure in the same plane is preferred can be answered by the following arguments: in the zigzag geometry, the strength of two $\sigma$-bonds formed between two nearest atoms (one due to a s-s orbital combination, the second due to a $\mathrm{p}_{x}-\mathrm{p}_{x}$ or $\mathrm{p}_{y}-\mathrm{p}_{y}$ combination) is maximized in the planar zigzag geometry for $\theta=90^{\circ}$. With the participation of the $\pi$-bond due to the $\mathrm{p}_{z}-\mathrm{p}_{z}$ orbital combination, triple bonds between adjacent zigzag atoms maintain the cohesion of the zigzag chain. In this way the two fold coordinated zigzag chain can acquire a cohesion slightly smaller, but comparable to, that of the three folded $2 \mathrm{D}$ monolayer of the same element (see Table 1). Additionally, in the zigzag geometry ten electrons per unit cell make five filled bands in the first zone; the highest band being fully filled and disjointed from the empty sixth band constituting a structure, which is more stable than an odd number of electrons with a half-filled band.

Notably, while the P-ZZ and As-ZZ chains are semimetals, their energetically second favorable AC-ZZ chains are semiconductors with direct band gaps of $0.38 \mathrm{eV}$ and $0.41 \mathrm{eV}$ at the $\Gamma$ point. As for the effect of the applied uniaxial strain on the energy band structure, upon the expansion of the lattice constant $a$ up to $10 \%$ the semimetallic characters of P-ZZ and As-ZZ are unaltered. However, under $4 \%$ uniaxial tensile strain, the band gaps of Sb-ZZ and Bi-ZZ increased to $96 \mathrm{meV}$ and $9 \mathrm{meV}$, respectively. Nevertheless, based on the above arguments, $\theta$ exceeding a threshold angle $\theta>90^{\circ}$ should open a gap.

The contributions of s- and $\mathrm{p}_{x, y, z}$-orbitals to the bands and to the PDOSs in Fig. 4(a) are in compliance with the above discussion. Notably, bands slightly flattened at the $\Gamma$-point construct sharp peaks.

Fig. 4(c) shows the isosurface of the total charge density and its contour plot, as well as the difference charge density obtained by subtracting the free atom densities from the total charge density of P-ZZ, as an example. The contour plot indicates high charge density along zigzag P-P-P bonds, unveiling the covalent character of the bonding. In addition, the $\pi$-bonds constructed 

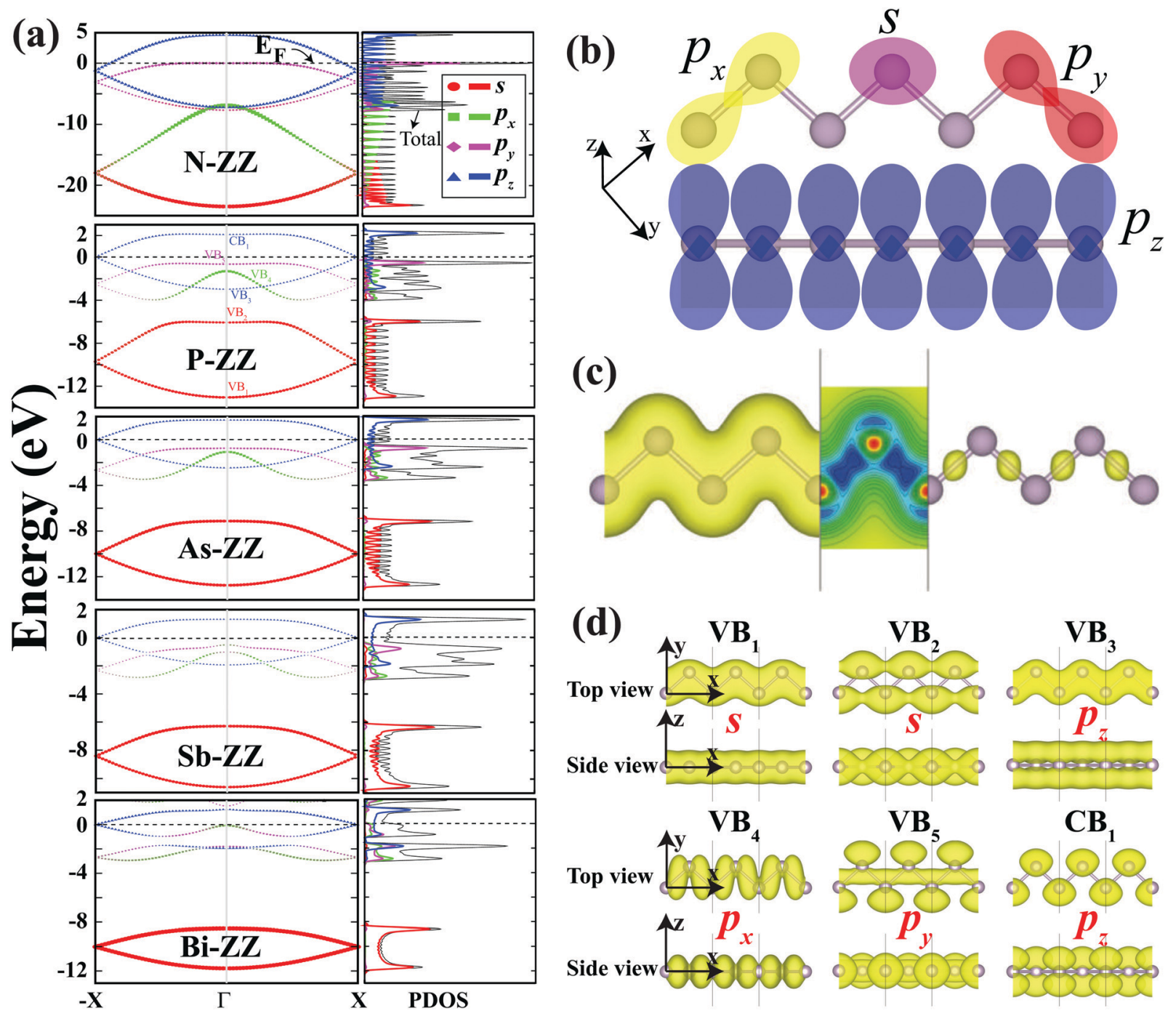

Fig. 4 (a) Electronic band structures and partial densities of states (PDOS) of the ZZ chains. Contributions of $p_{x^{-}}, p_{y^{-}}, p_{z^{-}}$and $s^{-}$orbitals to the bands, as well as to the DOS are indicated. The Fermi level is set to zero energy. The ZZ-chain is in the $x y$-plane and the $z$-axis is perpendicular to it. (b) Schematic orbital assignment to form bonds and bands of the ZZ-chain. (c) The total charge density $\rho_{\top}$ isosurface on the left and its contour plot showing the bond formation in the middle. The isosurface of the charge density difference, $\Delta \rho$, on the right shows the bond charges. (d) Top and side views of the charge density isosurfaces of band states of $\mathrm{P}-\mathrm{ZZ}$ at the center of the BZ are presented as an example. Band states are indicated in (a).

from bonding $\mathrm{p}_{z}$-orbitals contribute to the binding. The bond charges in the isosurfaces of the difference charge density on the right side are clearly seen.

In Fig. 4(d) the orbital characters of all the valence bands and the lowest conduction band of P-ZZ are presented. The first and second valence bands at the $\Gamma$-point are derived from the bonding and antibonding combinations of s-orbitals, i.e., $\sigma$ - and $\sigma^{*}$-bonding states, respectively. The third valence band is constructed from the bonding $(\pi)$ combination of $\mathrm{p}_{z}$-orbitals perpendicular to the plane of the chain. The fourth and fifth valence bands are constructed from the combination of $\mathrm{p}_{x}$ and $\mathrm{p}_{y}$-orbitals. The lowest conduction band consists of the antibonding combination of $\mathrm{p}_{z}$-orbitals, specified as $\pi^{*}$ bonding. These assignments of orbitals to the bands are in compliance with the above simple description regarding the band formation, as well as with the order of the bands of P-ZZ in Fig. 4(a).

\subsection{Zigzag chains supported by substrates}

Having revealed the dynamically and thermally stable structures, we next address the frequently asked question of whether these free-standing chain structures can occur on selected substrates and keep their physical properties. Earlier, strong hybridization effects have been revealed for a 2D silicene monolayer grown on the $A g(111)$ surface, modifying the electronic structure of the free-standing silicene. ${ }^{72}$ Similar effects have been found for a 1D structure of phosphorus on the $\mathrm{Au}(111)$ surface, whereby the formation of the well-defined zigzag chain was hindered. ${ }^{34}$ It is therefore important to know whether the properties predicted above for the free-standing chains can be modified when they are grown on specific substrates. To this end, we first examined zigzag monatomic chains placed on their parent 2D monolayers in hexagonal buckled honeycomb (hb) structures; namely P-ZZ on blue phosphorene, As-ZZ on hb-arsenene, Sb-ZZ on hb-antimonene 

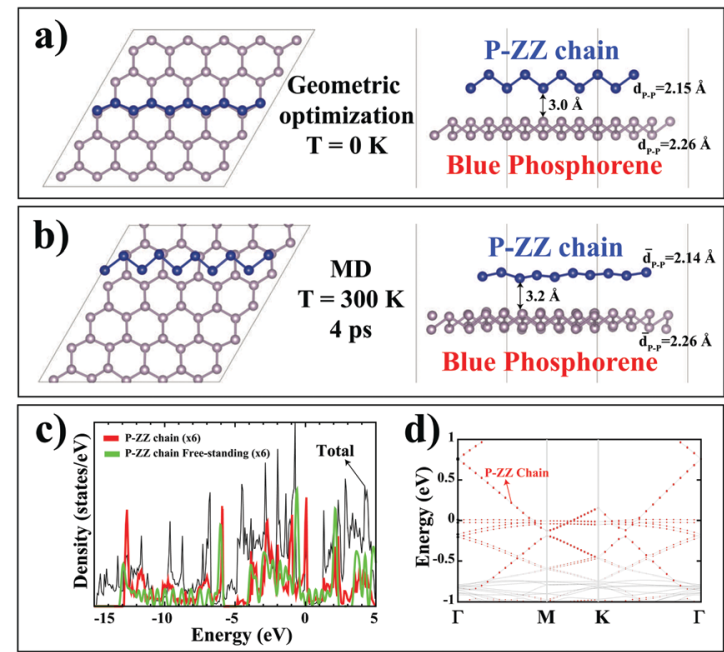

Fig. 5 (a) Top and side view of optimized P-ZZ supported by blue phosphorene. (b) Top and side views of snapshots of AIMD calculations on (a). (c) Total (TDOS) densities of states of the supported P-ZZ + blue phosphorene substrate, local density of states (LDOS) at the supported $\mathrm{P}-\mathrm{ZZ}$. The TDOS of free-standing P-ZZ is also shown for the sake of comparison. (d) Energy band structure of the supported $P-Z Z+$ blue phosphorene substrate. Red lines and circles indicate the contribution of the supported P-ZZ to the energy bands.

and Bi-ZZ on hb-bismuthenene. In addition, we examined P-ZZ on graphene, h-BN and GaSe monolayers. Our results are summarized in Fig. 5 and 6, and also in Fig. S1-S5 in the ESI. $\uparrow^{73}$

We found that P-, As-, Sb- and Bi-ZZ chains of pnictogen atoms can be stable on their parent $2 \mathrm{D}$ hb-monolayers. We also tested the stability of P-ZZ chains placed on graphene, h-BN and GaSe monolayers. First, the structures of the chains on these substrates are optimized at $T=0 \mathrm{~K}$, and their zigzag planes generally became tilted at a large distance from the substrate. This configuration indicates that the interaction between the chain and the supporting substrate is weak and composed of weak chemical and van der Waals attraction. The stability of these supported chains was assured by thermal stability analysis by performing AIMD calculations. Supported monatomic chains kept their zigzag structure on the substrate at $T=300 \mathrm{~K}$, except minute deformations induced by thermal excitations. The state densities shown in Fig. 5(c) indicate that the electronic properties of the free-standing P-ZZ chain are maintained when supported by blue phosphorene. This conclusion is arrived at by comparing the LDOS on the supported chain with the DOS of the freestanding chain, which appear to be similar. In Fig. 5(d) the metallic bands in the gap of blue phosphorene are constructed mainly by the P-ZZ chain. Accordingly, these chains, weakly bound to supporting substrates, constitute 1D metallic channels as interconnects between two semiconducting zones. One sees also similar behaviors in Fig. 6 for P-ZZ chains on a h-BN monolayer. In this case a narrow band gap is opened at the Fermi level, since the band crossing is not allowed by symmetry. Nevertheless, the behaviors observed in Fig. 5 repeat themselves with minute deviations in other pnictogen chains placed on their parent monolayers (namely As-ZZ on hb-arsenene, Sb-ZZ on
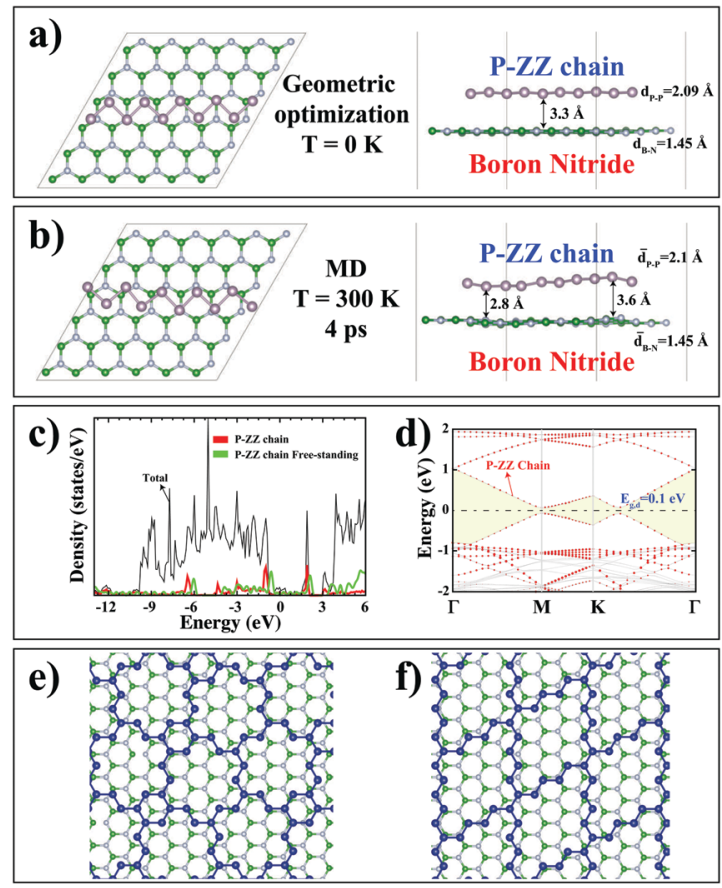

Fig. 6 (a) Top and side view of optimized P-ZZ supported by a h-BN monolayer. (b) Top and side views of snapshots of AIMD calculations on (a). (c) Total densities of states (TDOS) of the supported P-ZZ $+\mathrm{h}-\mathrm{BN}$ monolayer as the substrate and the local density of states (LDOS) at the supported P-ZZ. (d) Energy band structure of the supported P-ZZ + h-BN monolayer as the substrate. Red lines and circles indicate the contribution of the supported PP-ZZ to the energy bands. (e) A grid made by small hexagons and large coronone, and (f) parallelograms constructed by $\mathrm{P}-\mathrm{ZZ}$ on a $\mathrm{h}$-BN monolayer forming a metal-insulator framework ( $\mathrm{P}$ atoms highlighted blue).

hb-antimonene, and Bi-ZZ on hb-bismuthene) or other monolayers (P-ZZ on graphene, and on GaSe) as seen in the $\mathrm{ESI}^{73}$ (Fig. S1-S5). Notably, for Sb-ZZ on hb-antimonene and Bi-ZZ on hb-bismuthene, the separation between the chain and substrate is reduced, but the chain-substrate interaction is still weak due to reduced coordination. Owing to weak chain-substrate interaction, it is also expected that the electronic structure of finite size free-standing pnictogen chains is preserved when they are placed on substrates.

We note that not only stable monoatomic chains of P-ZZ weakly bound to the underlying monolayer, but also their artificial grids or networks of desired geometry can be constructed on the insulating substrates to form metal-insulator frameworks as shown in Fig. 6(e and f). Depending on the symmetry of the grid and the substrate, the band gap derived from P-ZZ can be tuned, and even be closed.

\subsection{Formation of supported and free-standing chains}

Another frequently raised question pertaining to atomic chains is whether they can really be produced or if they are only a sole theoretical prediction. Here we present conclusive clues attained from theoretical results that atomic chains of pnictogens can, in principle, be produced. In Fig. 7(a and b), P atoms randomly placed on a graphene monolayer form segments of P-ZZ upon 

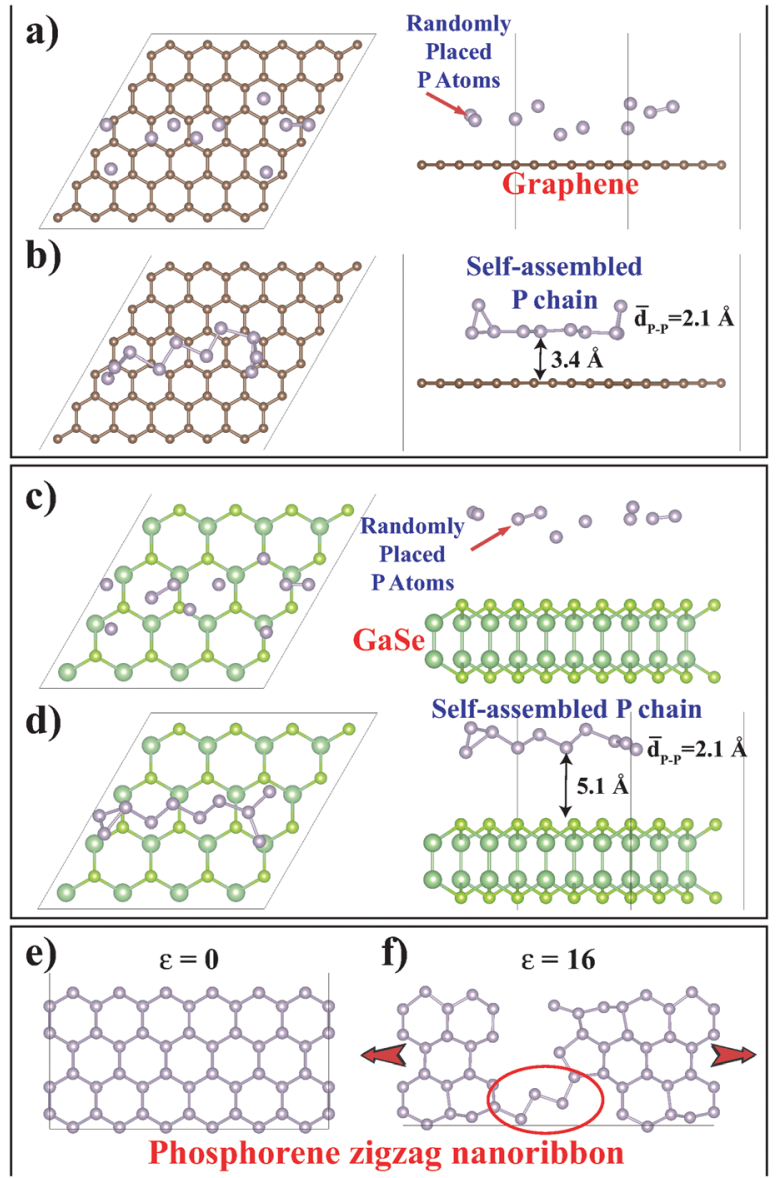

Fig. 7 (a) Initial top and side views of individual $P$ atoms randomly placed on a graphene $(6 \times 6)$ substrate. (b) Top and side views of self-assembled $P$ atoms forming zigzag chains on a graphene $(6 \times 6)$ substrate after relaxation. (c) Initial top and side views of individual $P$ atoms randomly placed on a GaSe $(4 \times 4)$ substrate. (d) Top and side views of self-assembled $P$ atoms forming zigzag chains on a GaSe $(4 \times 4)$ substrate after relaxation. (e) Strain free $(\varepsilon=0)$ phosphorene zigzag nanoribbon. (f) A segment of suspended P-ZZ is formed in this nanoribbon under a uniaxial tensile strain of $\varepsilon=0.16$.

relaxation. Similarly, $\mathrm{P}$ atoms randomly placed on a GaSe monolayer formed segments of P-ZZ as shown in Fig. 7(c and d). The structure of these supported atomic chains can further be improved by thermal relaxation. Also, Fig. 7(e and f) show that a zigzag nanoribbon of blue phosphorene with a vacancy defect can change to include a segment of a free-standing P-ZZ chain under high, uniaxial tensile strain. Notably, this conclusion is corroborated by the recent synthesis of narrow phosphorene nanoribbons. ${ }^{63}$ Similar monatomic gold chains were also produced in breakjunctions. While supported and free-standing zigzag monatomic chains of phosphorus were considered here as a proof of concept, we believe that monatomic chains of other pnictogens can also be formed.

\subsection{Electronic and magnetic properties of the finite zigzag monatomic chains}

Here we investigate the zigzag monatomic chains including a finite number, $n$, of group-VA elements. We considered $9 \leq$ $n \leq 19$, and examined the electronic and magnetic properties as a function of $n$. All finite chains are treated within a supercell geometry with lattice constants $a=40 \AA$ and $b=c=16 \AA$ to minimize the interchain interaction. The finite size brings about additional stability since long wavelength phonons, which are prone to instability, are eliminated. Upon structure optimization, all chains neither dissociated, nor formed clusters. Our results concerning the atomic structure and magnetic moments of finite chains of $\mathrm{P}, \mathrm{As}, \mathrm{Sb}$, and $\mathrm{Bi}$ are summarized in Fig. 8(a and b). Notably, finite size, zigzag chains of nitrogen differ from the finite chains of other group-VA elements. For example, while three $\mathrm{N}$ atoms at the ends of the chain tend to line up, finite size chains of all other group-VA elements keep the zigzag geometry even at the ends of the chain. This situation originates from the tendency to form the strong $\mathrm{N}_{2}$ molecule. Additionally, all finite size chains of nitrogen are nonmagnetic no matter what the value of $n$ is.

The atomic, electronic and magnetic structure of the finite zigzag chains of other pnictogens are dramatically different.
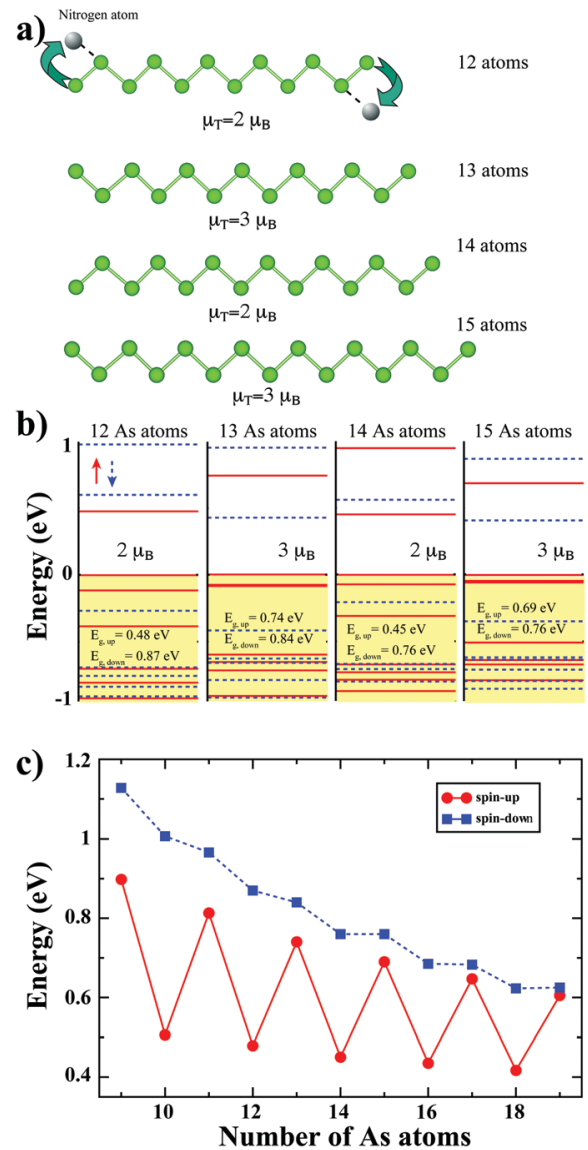

Fig. 8 (a) Prototype configurations of finite size, monatomic ZZ chains of group-VA elements which include 12-15 atoms. As an exception, the atomic configuration depicted by gray balls describes the line up of three end $\mathrm{N}$ atoms in finite size, $\mathrm{N}-\mathrm{ZZ}$ chains which are nonmagnetic. (b) Electronic energy states of the finite zigzag chains of As. The highest occupied molecular orbital (HOMO) is set to zero energy. Occupied states are shaded yellow. Blue dashed lines represent spin down states, while red solid lines are for spin up states. (c) Variation of the HOMO-LUMO band gaps of spin-up and spin-down states of finite size As-ZZ chains with respect to the number of As atoms. 
To determine the net magnetic moment, $\mu_{\mathrm{T}}$, of a $\mathrm{ZZ}$ finite chain having $n$ atoms $(n=9-19)$, we examined the magnetic moment on each atom calculated using the plane wave basis set within periodic boundary conditions. We found $\mu_{\mathrm{T}} \sim 3 \mu_{\mathrm{B}}$ for odd $n \mathrm{ZZ}$ chains of $\mathrm{P}, \mathrm{As}, \mathrm{Sb}$ and $\mathrm{Bi}$. However, our analysis shows that the total magnetic moment of P-ZZ, As-ZZ and Sb-ZZ finite chains with even $n$ is $\mu_{\mathrm{T}}=2 \mu_{\mathrm{B}}$, whereas Bi-ZZ chains with even $n$ have $\mu_{\mathrm{T}}=4 \mu_{\mathrm{B}}$. The majority of the magnetic moments are accumulated at the end atoms. These results are reproduced also by using Siesta software, which uses local basis sets. We believe that this is an important result that finite size $\mathrm{ZZ}$ monatomic chains of $\mathrm{P}$, As, $\mathrm{Sb}$ and $\mathrm{Bi}$ have a net magnetic moment, which depends on whether the number of chain atoms is even or odd. This is an interesting even-odd disparity.

In contrast to the periodic $\mathrm{ZZ}$ chains, finite chains have a HOMO-LUMO gap. In Fig. 8(b) the electronic energy level structures of finite As-ZZ are shown for $n=12-15$, where zero energy is set to the energy of the HOMO of the spin up states, which is always the highest occupied orbital. In Fig. 8(c), we present the variation of the HOMO-LUMO band gaps of the spin up and spin down states of As-ZZ as a function of the number $n$ of atoms. We note that for all $n$, the HOMO-LUMO gap of the spin up states is smaller than that of the spin down states. Notably, the lowest LUMO energy alternates between spin up and spin down states depending on an even/odd value of $n$. For example, while the lowest energy LUMO corresponds to a spin up state for even $n$, it changes to a spin down state for odd $n$. This is also closely related with the even-odd disparity in the magnetic moment of the chain. The HOMO-LUMO band gaps vary with $n$ for both spin up and spin down states. They also decrease gradually with increasing $n$ as a manifestation of the confinement effect. However, the difference of the HOMOLUMO band gap between $n$ and $n+1$ chains is enhanced for spin-up states with decreasing $n$. This originates from the significant shift of the energy of the spin up LUMO depending on even/odd values of $n$ and hence is related to the space symmetry of the LUMO.

\subsection{Periodic chains of group-VA binary compounds}

Earlier theoretical studies predicted that 2D monolayers of binary compounds of group-VA elements are dynamically and thermally stable materials. ${ }^{74-77}$ Additionally, strain induced quantum spin Hall insulators ${ }^{52}$ and giant and tunable Rashba spin splitting ${ }^{53}$ from monolayers of SbBi binary compounds in buckled hexagonal structures have been predicted. Li et al. showed that $\mathrm{Bi}(110)$ films $^{78}$ and amidogen modified $\mathrm{Bi} / \mathrm{Sb}(111)$ films $^{79}$ are ideal platforms of the QSH effect for low-power dissipation devices. While interesting and fundamental effects were unveiled for 2D SbBi binary compounds, the chains of binary compounds of group-VA elements gained importance. In this section, we examined periodic chains of group-VA compounds in the $\mathrm{ZZ}$ structures. We considered four compound chains, namely NP-ZZ, PAs-ZZ, AsSb-ZZ and SbBi-ZZ. Similar to the monatomic chains, we first performed tests of the dynamical and the thermal stabilities of these compounds. Fig. 9 illustrates the phonon dispersions and snapshots of AIMD simulations carried out at $T=500 \mathrm{~K}$ taken after $4 \mathrm{ps}$.

Out of four periodic chains, one acoustic mode of NP-ZZ appears to have imaginary frequencies. We believe that it might be due to a numerical accuracy problem, instead of a dynamical instability. This conclusion is corroborated by the fact that NP-ZZ does not dissociate in AIMD carried out at $T=500 \mathrm{~K}$ after 4 ps simulation. Nonetheless, as depicted in the bottom panel of Fig. 9, the deformation of NP-ZZ at $T=500 \mathrm{~K}$ is severe as compared to the other compound chains. This suggests that NP-ZZ will be prone to instability for $T>500 \mathrm{~K}$. Except for the
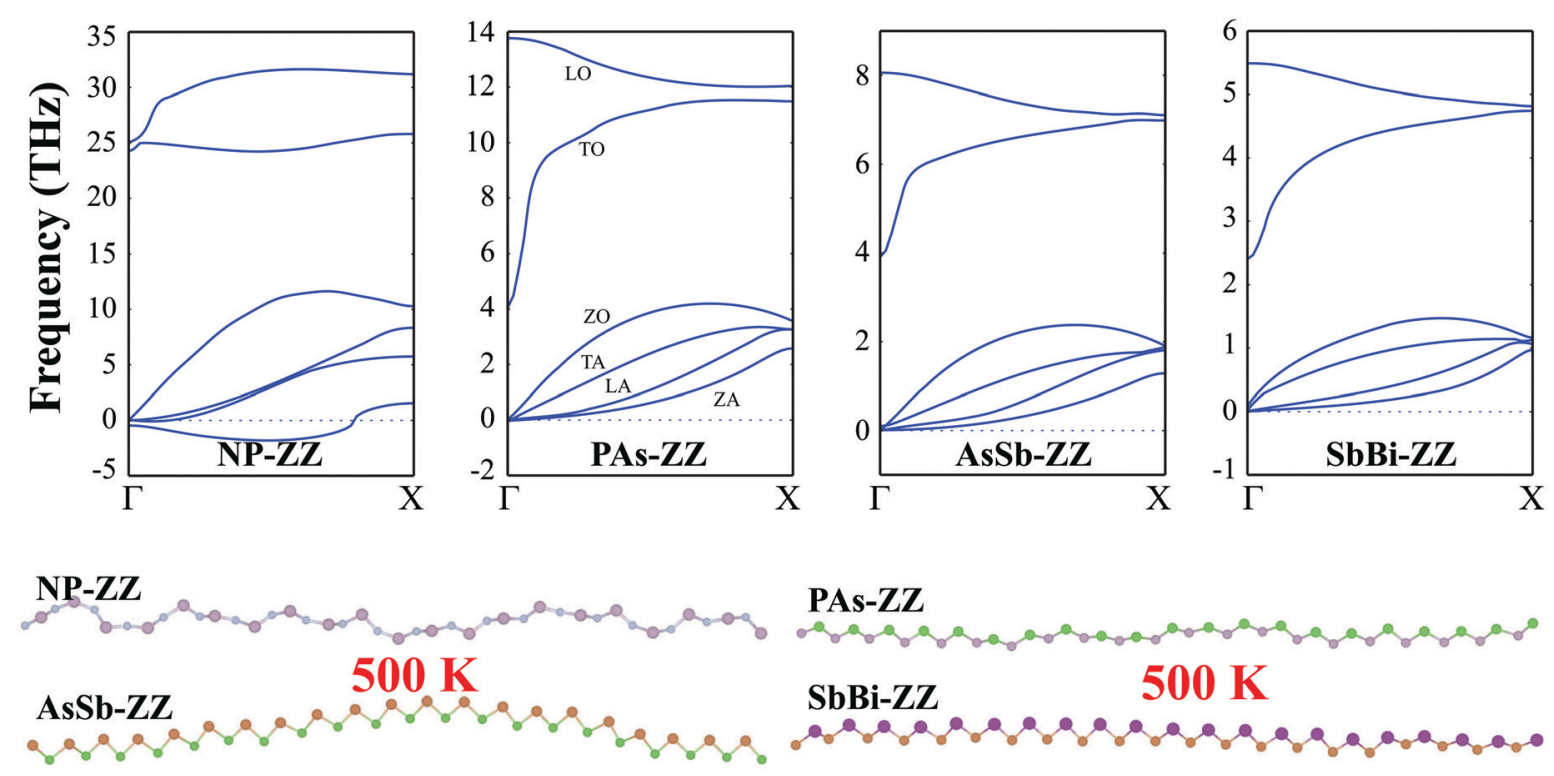

PAs-ZZ

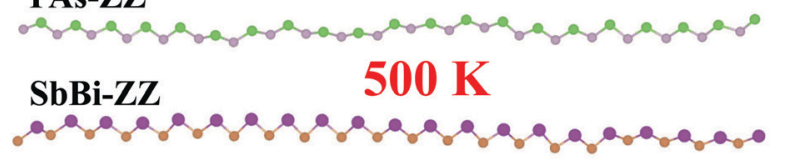

Fig. 9 Periodic chains of group-VA compounds. Top: Phonon dispersions for $\mathbf{k}$ varying along the axis of the chain. Bottom: Snapshots of AIMD simulations at $T=500 \mathrm{~K}$ after $4 \mathrm{ps}$. 
Table 2 Structural parameters and energetics calculated for the periodic, zigzag chains of binary compounds of group-VA elements. Lattice parameter (a); bond length $\left(d_{1,2}\right)$; bond angle $(\theta)$; cohesive energy $\left(E_{c}\right)$; and band gaps $E_{g}$, calculated by PBE + SOC and HSE + SOC, d or i in right parenthesis indicates a direct or indirect band gap

\begin{tabular}{lllllll}
\hline VV compound & $a(\AA)$ & $d_{\mathrm{V}-\mathrm{V}}(\AA)$ & $\theta($ degree $)$ & $E_{\mathrm{c}}(\mathrm{eV}$ per atom $)$ & $E_{\mathrm{g}}(\mathrm{PBE}+\mathrm{SOC})(\mathrm{eV})$ & $E_{\mathrm{g}}(\mathrm{HSE}+\mathrm{SOC})(\mathrm{eV})$ \\
\hline NP-ZZ & 2.62 & 1.64 & 106.5 & 4.01 & $1.18(\mathrm{i})$ & $0.15(\mathrm{~d})$ \\
PAs-ZZ & 3.32 & 2.25 & 95.1 & 2.78 & $0.25(\mathrm{~d})$ & $0.38(\mathrm{i})$ \\
AsSb-ZZ & 3.73 & 2.56 & 93.6 & 2.43 & $0.05(\mathrm{~d})$ & $0.71(\mathrm{~d})$ \\
SbBi-ZZ & 4.06 & 2.84 & 91.2 & 2.47 & $0.17(\mathrm{~d})$
\end{tabular}

NP-ZZ chain, the highest (longitudinal optical (LO)) and the second highest (transverse optical (TO)) vibrational optical modes are separated from each other at the $\Gamma$-point, but they become closer at the $X$ point for all compound chains.

The compound chains gain kinetic energy with increasing temperature and rotate around their axis, keeping their zigzagpattern. Calculated structural parameters and energies of the optimized compound chains are given in Table 2. As can be seen, the cohesive energy values of the compound chains are slightly higher than the average value of the cohesive energy of the constituent 2D monatomic chains. Notably, these cohesive energy values of the compound chains are almost half of the cohesive energy of the corresponding $2 \mathrm{D}$ monolayer compounds $\mathrm{s}^{74,77}$ due to the lower number of coordination. In contrast to the $\mathrm{ZZ}$ monatomic chains of group-VA elements, all binary compound chains are semiconductors as shown in Fig. 10. According to PBE + SOC calculations, they are direct, narrow band gap semiconductors, except NP-ZZ, which has an indirect band gap of $1.18 \mathrm{eV}$. The direct band gaps of the other chains are rather narrow. In particular, the band gap of SbBi-ZZ is only $50 \mathrm{meV}$ and the dispersion of the highest valence band along
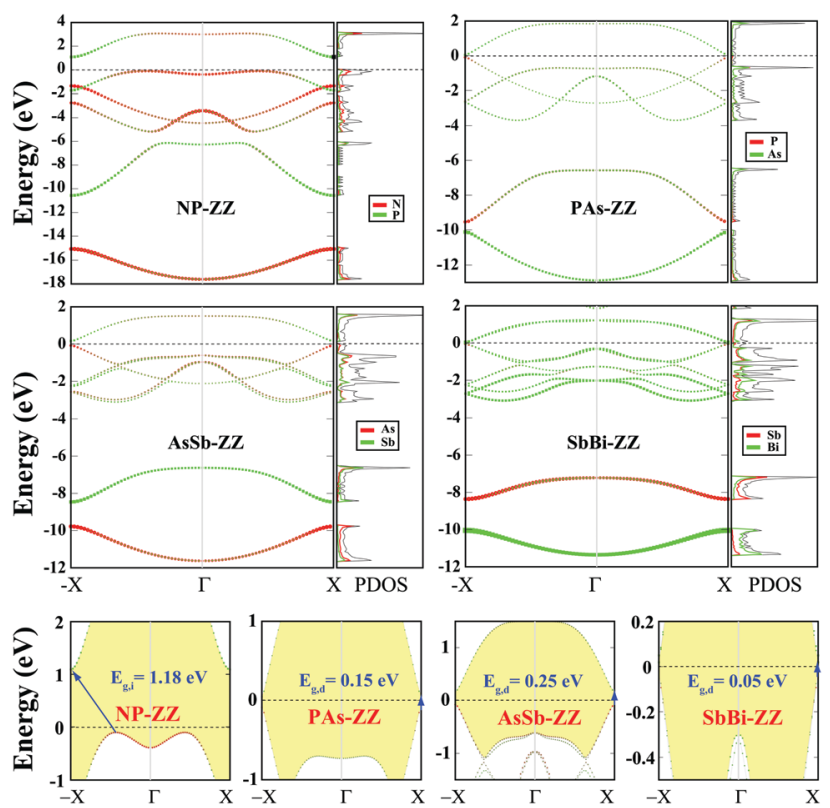

Fig. 10 Electronic energy band structures and partial densities of states (PDOS) of periodic, zigzag chains of group-VA binary compounds. The contributions of the constituent elements to various bands are depicted by the size and color of dots. The electronic structures near the Fermi level are zoomed in in the bottom panels. The Fermi level is set to zero energy. the $\Gamma-X$ direction exhibits peculiarities. In this respect, the electronic structure of the SbBi-ZZ chain deserves detailed analysis in view of possible topological behaviors.

\subsection{Adsorption of adatoms to zigzag chains}

The properties of nanostructures like atomic chains can be modified for functionalization by the adsorption of adatoms or molecules. Adatoms can readily be adsorbed to the monatomic chains. However, owing to their lower dimensionality, the chain structure may be dissociated under high coverage of reactive adatoms, which weaken the bonds in the chain. For example, full coverage corresponding to the adsorption of one adatom per host atom can lead to dissociation. In the present study, we considered adsorption of $\mathrm{O}$ and $\mathrm{H}$ atoms and $\mathrm{OH}$ (hydroxy) groups to the periodic, zigzag, monatomic chains. We first considered full coverage $(\theta=1)$ of $\mathrm{O}$ atoms for all periodic zigzag chains. At full coverage of $\mathrm{O}$ atoms on all periodic, zigzag chains, the chain structures are destroyed. Moreover, As-ZZ, $\mathrm{Sb}-\mathrm{ZZ}$ and Bi-ZZ are also dissociated at full coverage of $\mathrm{H}$ and $\mathrm{OH}$. Interestingly the P-ZZ chain sustained and kept its zigzag geometry at full coverage of $\mathrm{H}$ or $\mathrm{OH}$. Since the bond energy of $\mathrm{P}-\mathrm{ZZ}$ is rather high and $3.10 \mathrm{eV}, \mathrm{P}-\mathrm{P}$ bonds cannot be broken with the formation of the $\mathrm{H}-\mathrm{P}, \mathrm{OH}-\mathrm{P}$ and $\mathrm{HO}-\mathrm{P}$ bonds at full coverage. In Table 3 we present the calculated values of the binding energy, $E_{\mathrm{b}}$, and corresponding structural parameters for $\mathrm{H}, \mathrm{O}$ and $\mathrm{OH}$ adsorbed onto $\mathrm{P}-\mathrm{ZZ}$ at full coverage. Notably, $\mathrm{O}$ atom binding to $\mathrm{P}-\mathrm{ZZ}$, which is stronger than the P-P bond of bare P-ZZ, induces instability at full coverage. As a test case, we considered various coverage values of $\mathrm{O}$ atoms adsorbed onto the Bi-ZZ chain and found that the chain sustained oxidation corresponding to $\theta \leq 1 / 6$. Even if the Bi chain does not dissociate under low $\mathrm{O}$ coverage, the Bi-ZZ chain undergoes a reconstruction; the zigzag angle becomes wider at the adsorption site of $\mathrm{O}$. We note that the adsorption of $\mathrm{H}$ and $\mathrm{OH}$ can modify the electronic band structure of the P-ZZ chain. While full

Table 3 Structural parameters and binding energies of selected adatoms and molecules adsorbed onto the periodic P-ZZ chain; lattice parameter $(a)$, bond length $\left(d_{P-P}\right)$ between host $P$ atoms, distance between the adsorbate $(X)$ and the atom in the chain $\left(d_{\mathrm{p}-\mathrm{X}}\right)$, and binding energy $\left(E_{\mathrm{b}}\right)$

\begin{tabular}{lllll}
\hline & $\mathrm{H}-\mathrm{P}$ & $\mathrm{O}-\mathrm{P}$ & $\mathrm{OH}-\mathrm{P}$ & $\mathrm{HO}-\mathrm{P}$ \\
\hline$a(\AA)$ & 3.15 & 3.15 & 3.15 & 3.14 \\
$\theta$ (degree) & 97.1 & 100.1 & 91.9 & 96.0 \\
$d_{\mathrm{V}-\mathrm{V}}(\AA)$ & 2.10 & 2.06 & 2.19 & 2.11 \\
$d_{\mathrm{V}-\mathrm{X}}(\AA)$ & 1.53 & 1.49 & 1.65 & 2.64 \\
$E_{\mathrm{b}}(\mathrm{eV}$ per adatom) & 0.83 & 4.77 & 1.89 & 0.06
\end{tabular}


coverage of hydroxyl groups opens the band gap to $70 \mathrm{meV}$, hydrogen coverage turns the semimetal P-ZZ chain to a metal.

\section{Discussions and conclusions}

Our study on the monatomic chains of pnictogens unveiled features which are crucial for systems of lower dimensionality from both fundamental and technological points of view. Among diverse atomic chain models we found that the periodic zigzag chain geometry is the most stable and lowest energy configuration for group-VA elements $\mathrm{P}, \mathrm{As}, \mathrm{Sb}$ and Bi. Nitrogen being an exception, it has the lowest energy configuration for the linear, Peierls dimerized model due to the fact that the $\sigma$ and $\pi$-bonding of $\mathrm{p}_{x, y, z}$ orbitals become comparable at small bond length. However, this linear chain geometry is prone to dynamical instabilities, since it cannot overcome the formation energy of an $\mathrm{N}_{2}$ molecule. Using a bond orbital model we explained why the zigzag chain model with a bond angle of $\theta \sim 90^{\circ}$ provides the strongest bonding. This model also explains the character and orders of the bands derived from these bonds. According to this model, the half-filled band is folded at the 1D zone boundary upon the formation of the zigzag structure, forming five filled valence bands and empty conduction bands. Normally, the highest filled band crosses the empty band at the zone boundary linearly. However, owing to spin orbit coupling combined with the deviations of the bond angle from $90^{\circ}$ degrees the linearly crossing bands split and open a very narrow band gap. While the electronic band structures of zigzag monatomic chains of $\mathrm{P}, \mathrm{As}, \mathrm{Sb}$ and $\mathrm{Bi}$ comply with this interpretation, the empty band of the zigzag chain of nitrogen overlaps with the up shifted $\mathrm{p}_{y}$-band and hence becomes metallized. In this way, periodic zigzag chains of nitrogen become prone to instability at the long wavelength limit. The 1D "mexican hat" form of the highest filled band of the zigzag Bi chain is of particular interest because of the possible new quantum states it can attain. Similarly, the form of the highest bands of zigzag Sb chains, which is very close to band inversion, can be changed by external effects like axial strain or an axial electric field to transform to topologically interesting states.

When placed on their parent 2D monolayers or monolayers like graphene, h-BN and GaSe, monatomic chains of pnictogens have weak interaction with the underlying substrate and remain stable. These supported monatomic chains preserve their character attained when they were free-standing. Long monatomic pnictogen chains can produce 1D metallic channels on 2D semiconducting substrates and act as metallic interconnects. They can be utilized as quantum switches. The grids of these monatomic chains constructed on the insulating monolayers can form 1D/2D composite structures or metal-insulator frameworks of desired geometries offering diverse functionalities.

The finite size, P, As, Sb and Bi zigzag chains preserved their zigzag geometry, but attained finite magnetic moments. The chains having an even number of atoms had a magnetic moment of two Bohr magnetons. Whereas those chains comprising odd numbers of atoms gained three Bohr magnetons. Additionally, the HOMO-LUMO gaps of spin up and spin down states exhibit interesting alternations depending on the even/odd values of the numbers of chain atoms and offer critical tunability of the electronic structure. We believe that the predicted even-odd disparity in magnetic states can have interesting implications. For example, one can monitor the quantized conductance of different spin currents with the magnitude of the applied axial electric field. Notably, all finite size zigzag nitrogen chains are found to be nonmagnetic due to the line up of three end atoms at both sides.

Not only monatomic chains but also binary compound zigzag chains of pnictogen atoms are predicted to be stable. However, owing to different potentials of the different constituent atoms forming the chain a small gap opens at the zone boundary, which can be tuned also by increasing the difference of row numbers of the constituent elements. However, these band gaps can be changed by electric fields and strain. In particular, in the binary compound zigzag chains of $\mathrm{Sb}$ and $\mathrm{Bi}$, the maximum of the valence band at the center of the Brillouin zone may offer a band inversion to lead to interesting quantum states as revealed in $3 \mathrm{D}$ and $2 \mathrm{D}$ compounds of the same elements.

Full adsorption on the chains of foreign atoms like $\mathrm{H}$ and $\mathrm{O}$ and $\mathrm{OH}$ groups is also investigated. All zigzag chains except the chain of $\mathrm{P}$ have dissociated at full coverage of $\mathrm{H}$ and $\mathrm{OH}$. In the functionalization of chains with foreign atoms the strength of the bond between the host pnictogen and the foreign atom appears to be decisive. It appears that zigzag chains of As, Sb and $\mathrm{Bi}$ can remain stable when they covered with less reactive atoms at dilute coverage.

As a final remark, based on theoretical simulations we demonstrated that free-standing and supported pnictogen zigzag monatomic chains can be produced. The diverse features of these chains revealed in this study are fundamental insofar as they are complementary to other lower dimensional allotropes, like monolayers and multilayers of group-VA elements. In addition, the physical properties predicted for these free chains offer future applications. In this respect, the investigation of the axial strain and electric field, pnictogen point impurities in monatomic chains and their effects can be interesting for future studies.

\section{Conflicts of interest}

There are no conflicts to declare.

\section{Acknowledgements}

The computational resources were provided by TUBITAK ULAKBIM, High Performance and Grid Computing Center (TR-Grid e-Infrastructure). S. C. acknowledges financial support from the Academy of Sciences of Turkey (TUBA).

\section{References}

1 K. S. Novoselov, A. K. Geim, S. V. Morozov, D. Jiang, M. I. Katsnelson, I. V. Grigorieva, S. V. Dubonos and A. A. Firsov, Two-dimensional gas of massless Dirac fermions in graphene, Nature, 2005, 438, 197. 
2 N. D. Lang and P. Avouris, Oscillatory Conductance of Carbon-Atom Wires, Phys. Rev. Lett., 1998, 81, 3515.

3 N. D. Lang and P. Avouris, Carbon-Atom Wires: ChargeTransfer Doping, Voltage Drop, and the Effect of Distortions, Phys. Rev. Lett., 2000, 84, 358.

4 A. Abdurahman, A. Shukla and M. Dolg, Ab initio many-body calculations on infinite carbon and boron-nitrogen chains, Phys. Rev. B: Condens. Matter Mater. Phys., 2002, 65, 115106.

5 S. Tongay, R. T. Senger, S. Dag and S. Ciraci, Ab initio Electron Transport Calculations of Carbon Based String Structures, Phys. Rev. Lett., 2004, 93, 136404.

6 E. Tekman and S. Ciraci, Novel features of quantum conduction in a constriction, Phys. Rev. B: Condens. Matter Mater. Phys., 1989, 39, 8772(R).

7 J. K. Gimzewski and R. Möller, Transition from the tunneling regime to point contact studied using scanning tunneling microscopy, Phys. Rev. B: Condens. Matter Mater. Phys., 1987, 36, 1284.

8 S. Ciraci and E. Tekman, Theory of transition from the tunneling regime to point contact in scanning tunneling microscopy, Phys. Rev. B: Condens. Matter Mater. Phys., 1989, 40, 11969.

9 A. I. Yanson, G. Rubio Bollinger, H. E. van den Brom, N. Agrait and J. M. van Ruitenbeek, Formation and manipulation of a metallic wire of single gold atoms, Nature, 1998, 395, 783.

10 N. Agrat, J. G. Rodrigo and S. Vieira, Conductance steps and quantization in atomic-size contacts, Phys. Rev. B: Condens. Matter Mater. Phys., 1993, 47, 12345.

11 H. Mehrez and S. Ciraci, Yielding and fracture mechanisms of nanowires, Phys. Rev. B: Condens. Matter Mater. Phys., 1997, 56, 12632.

12 D. Sanchez-Portal, E. Artacho, J. Junquera, P. Ordejon, A. Garcia and J. M. Soler, Stiff Monatomic Gold Wires with a Spinning Zigzag Geometry, Phys. Rev. Lett., 1999, 83, 3884.

13 H. Häkkinen, R. N. Barnett and U. Landman, Gold Nanowires and Their Chemical Modifications, J. Phys. Chem. B, 1999, 103, 8814.

14 P. Sen, S. Ciraci, A. Buldum and I. P. Batra, Structure of aluminum atomic chains, Phys. Rev. B: Condens. Matter Mater. Phys., 2001, 64, 195420.

15 S. Iijima and T. Ichihashi, Single-shell carbon nanotubes of 1-nm diameter, Nature, 1993, 363, 603-605.

16 E. Durgun, R. T. Senger, H. Mehrez, H. Sevincli and S. Ciraci, Size-dependent alternation of magnetoresistive properties in atomic chains, J. Phys. Chem., 2006, 125, 121102.

17 C. Jin, H. Lan, L. Peng, K. Suenaga and S. Iijima, Deriving Carbon Atomic Chains from Graphene, Phys. Rev. Lett., 2009, 102, 205501.

18 S. Cahangirov, M. Topsakal and S. Ciraci, Long-range interactions in carbon atomic chains, Phys. Rev. B: Condens. Matter Mater. Phys., 2010, 82, 195444.

19 S. Tongay, E. Durgun and S. Ciraci, Atomic strings of group IV, III-V, and II-VI elements, Appl. Phys. Lett., 2004, 85, 6179.

20 R. T. Senger, S. Tongay, E. Durgun and S. Ciraci, Atomic chains of group-IV elements and III-V and II-VI binary compounds studied by a first-principles pseudopotential method, Phys. Rev. B: Condens. Matter Mater. Phys., 2005, 72, 075419.

21 S. Dag, S. Tongay, T. Yildirim, E. Durgun, R. T. Senger, C. Y. Fong and S. Ciraci, Half-metallic properties of atomic chains of carbon-transition-metal compounds, Phys. Rev. B: Condens. Matter Mater. Phys., 2005, 72, 155444.

22 C. K. Yang, J. Zhao and J. P. Lu, Magnetism of transitionmetal/carbon-nanotube hybrid structures, Phys. Rev. Lett., 2003, 90, 257203.

23 C. Ataca, S. Cahangirov, E. Durgun, Y.-R. Jang and S. Ciraci, Structural, electronic, and magnetic properties of 3d-transition metal monatomic chains: first-principles calculations, Phys. Rev. B: Condens. Matter Mater. Phys., 2008, 77, 214413.

24 D. E. Angelescu, M. C. Cross and M. L. Roukes, Heat transport in mesoscopic systems, Superlattices Microstruct., 1998, 23, 673.

25 L. G. C. Rego and G. Kirczenow, Quantized Thermal Conductance of Dielectric Quantum Wires, Phys. Rev. Lett., 1998, 81, 232.

26 A. Buldum, S. Ciraci and C. Y. Fong, Quantum heat transfer through an atomic wire, J. Phys.: Condens. Matter, 2000, 12, 3349.

27 A. Ozpineci and S. Ciraci, Quantum effects of thermal conductance through atomic chains, Phys. Rev. B: Condens. Matter Mater. Phys., 2001, 63, 125415.

28 H.-J. Kim and J.-H. Cho, Giant spin-orbit-induced spin splitting in Bi zigzag chains on GaAs(110), Phys. Rev. B: Condens. Matter Mater. Phys., 2015, 92, 085303.

29 T. Hashizume, S. Heike, M. I. Lutwyche, S. Watanabe, K. Nakajima, T. Nishi and T. Wada, Interaction of $\mathrm{Ga}$ adsorbates with dangling bonds on the hydrogen terminated Si (100) surface, Jpn. J. Appl. Phys., 1996, 35, L1085.

30 A. Kida, H. Kajiyama, S. Heike, T. Hashizume and K. Koike, Self-organized growth of Fe nanowire array on $\mathrm{H}_{2} \mathrm{O} / \mathrm{Si}(100)$ $(2 \times n)$, Appl. Phys. Lett., 1999, 75, 540.

31 M. M. R. Evans and J. Nogami, Indium and gallium on Si(001): a closer look at the parallel dimer structure, Phys. Rev. B: Condens. Matter Mater. Phys., 1999, 59, 7644.

32 G. Shen and D. Chen, One-dimensional nanostructures for electronic and optoelectronic devices, Optoelectronics, 2010, $3(2), 125$.

33 Z. Xiao, J. Qiao, W. Lu, G. Ye, X. Chen, Z. Zhang, W. Ji, J. Li and C. Jin, Deriving phosphorus atomic chains from fewlayer black phosphorus, Nano Res., 2017, 10, 2519.

34 J.-P. Xu, J.-Q. Zhang, H. Tian, H. Xu, W. Ho and M. Xie, Onedimensional phosphorus chain and two-dimensional blue phosphorene grown on $\mathrm{Au}(111)$ by molecular-beam epitaxy, Phys. Rev. Mater., 2017, 1, 061002(R).

35 D. R. Bowler, C. F. Bird and J. H. G. Owen, 1D semiconducting atomic chain of In and $\mathrm{Bi}$ on $\mathrm{Si}(001)$, J. Phys.: Condens. Matter, 2006, 18, L241.

36 J. Javorský, J. H. Gervase Owen, M. Setvín and K. Miki, Electronic structure of Bi lines on clean and H-passivated Si(100), J. Phys.: Condens. Matter, 2010, 22, 175006.

37 V. Vierimaa, A. V. Krasheninnikov and H.-P. Komsa, Phosphorene under electron beam: from monolayer to onedimensional chains, Nanoscale, 2016, 8, 7949. 
38 J. Qiao, L. Zhou and W. Ji, Geometric stability and electronic structure of infinite and finite phosphorus atomic chains, Chin. Phys. B, 2017, 26, 036803.

39 H. Liu, A. T. Neal, Z. Zhu and Z. Luo, et al., Phosphorene: An Unexplored 2D Semiconductor with a High Hole Mobility, ACS Nano, 2014, 8, 4033.

40 L. Kou, C. Chen and S. C. Smith, Phosphorene: Fabrication, Properties, and Applications, J. Phys. Chem. Lett., 2015, 6, 2794.

41 V. O. Özçelik, O. U. Aktürk, E. Durgun and S. Ciraci, Prediction of a two-dimensional crystalline structure of nitrogen atoms, Phys. Rev. B: Condens. Matter Mater. Phys, 2015, 92, 125420.

42 O. U. Aktürk, V. O. Özçelik and S. Ciraci, Single-layer crystalline phases of antimony: antimonenes, Phys. Rev. B: Condens. Matter Mater. Phys., 2015, 91, 235446.

43 M. Ezawa, Monolayer Topological Insulators: Silicene, Germanene, and Stanene, J. Phys. Soc. Jpn., 2015, 84, 121003.

44 S. Zhang, Z. Yan, Y. Li, Z. Chen and H. Zeng, Atomically thin arsenene and antimonene: semimetal-semiconductor and indirect-direct band-gap transitions, Angew. Chem., 2015, $127,3155$.

45 E. Aktürk, O. U. Aktürk and S. Ciraci, Single and bilayer bismuthene: stability at high temperature and mechanical and electronic properties, Phys. Rev. B, 2016, 94, 014115.

46 F. Ersan, E. Aktürk and S. Ciraci, Stable single-layer structure of group-V elements, Phys. Rev. B, 2016, 94, 245417.

47 D. Kecik, E. Durgun and S. Ciraci, Stability of single-layer and multilayer arsenene and their mechanical and electronic properties, Phys. Rev. B, 2016, 94, 205409.

48 H. Guo, N. Lu, J. Dai, X. Wu and X. C. Zheng, Tuning Electronic and Magnetic Properties of Early TransitionMetal Dichalcogenides via Tensile Strain, J. Phys. Chem. C, 2014, 118, 14051.

49 M. Abid, A. Shoaib, M. H. Farooq, H. Wu, D. Ma and B. Fu, Edge magnetism and electronic structure properties of zigzag nanoribbons of arsenene and antimonene, J. Phys. Chem. Solids, 2017, 110, 167.

50 Y. Wang and Y. Ding, Electronic Structure and Carrier Mobilities of Arsenene and Antimonene Nanoribbons: A First-Principle Study, Nanoscale Res. Lett., 2015, 10, 254.

51 F. Ersan, D. Kecik, V. O. Ozcelik, Y. Kadioglu, O. U. Akturk, E. Durgun, E. Akturk and S. Ciraci, Two-dimensional pnictogens: A review of recent progresses and future research directions, Appl. Phys. Rev., 2019, 6, 021308.

52 W. Yu, C.-Y. Niu, Z. Zhu, X. Cai, L. Zhang, S. Bai, R. Zhao and Y. Jia, Strain induced quantum spin Hall insulator in monolayer $\beta$-BiSb from first-principles study, RSC Adv., 2017, 7, 27816.

53 S. Singh and A. H. Romero, Giant tunable Rashba spin splitting in a two-dimensional BiSb monolayer and in BiSb/ AlN heterostructures, Phys. Rev. B, 2017, 95, 165444.

54 G. Kresse and J. Furthmüller, Efficient iterative schemes for $a b$ initio total-energy calculations using a plane-wave basis set, Phys. Rev. B: Condens. Matter Mater. Phys., 1996, 54, 11169.
55 P. E. Blöchl, Projector augmented-wave method, Phys. Rev. B: Condens. Matter Mater. Phys., 1994, 50, 17953.

56 J. P. Perdew, K. Burke and M. Ernzerhof, Generalized Gradient Approximation Made Simple, Phys. Rev. Lett., 1996, 77, 3865.

57 S. Grimme, Semiempirical GGA-type density functional constructed with a long-range dispersion correction, J. Comput. Chem., 2006, 27, 1787.

58 H. J. Monkhorst and J. D. Pack, Special points for Brillouinzone integrations, Phys. Rev. B: Solid State, 1976, 13, 5188.

59 J. Heyd, G. E. Scuseria and M. Ernzerhof, Hybrid functionals based on a screened Coulomb potential, J. Chem. Phys, 2003, 118, 8207 (Erratum Hybrid functionals based on a screened Coulomb potential, J. Chem. Phys., 2006, 124, 219906).

60 J. Paier, M. Marsman, K. Hummer, G. Kresse, I. C. Gerber and J. G. Angyan, Screened hybrid density functionals applied to solids, J. Chem. Phys., 2006, 125, 249901.

61 J. M. Soler, E. Artacho, J. D. Gale, J. Junquera, P. Ordejon and D. Sanchez-Portal, The SIESTA method for $a b$ initio order-N materials simulation, J. Phys.: Condens. Matter, 2002, 14, 2745.

62 A. Togo and I. Tanaka, First principles phonon calculations in materials science, Scr. Mater., 2015, 108, 1.

63 M. C. Watts, L. Picco, F. S. Russell-Pavier, P. L. Cullen, T. S. Miller, S. P. Bartus, O. D. Payton, N. T. Skipper, V. Tileli and C. A. Howard, Production of phosphorene nanoribbons, Nature, 2019, 568, 216-220.

64 Y. Zhang, J. Lee, W. L. Wang and D.-X. Yao, Two-dimensional octagon-structure monolayer of nitrogen group elements and the related nano-structures, Comput. Mater. Sci., 2015, 110, 109-114.

65 Q. Wei and X. Peng, Superior mechanical flexibility of phosphorene and few-layer black phosphorus, Appl. Phys. Lett., 2014, 104, 251915.

66 C. Kamal and M. Ezawa, Arsenene: two-dimensional buckled and puckered honeycomb arsenic systems, Phys. Rev. B: Condens. Matter Mater. Phys., 2015, 91, 085423.

67 J. Carrete, L. J. Gallego and N. Mingo, Structural Complexity and Phonon Physics in 2D Arsenenes, J. Phys. Chem. Lett., 2017, 8, 1375-1380.

68 G. Wang, R. Pandey and S. P. Karna, Atomically Thin Group V Elemental Films: Theoretical Investigations of Antimonene Allotropes, ACS Appl. Mater. Interfaces, 2015, 7(21), 11490-11496.

69 L. Kou, X. Tan, Y. Ma, H. Tahini, L. Zhou, Z. Sun, D. Aijun, C. Chen and S. C. Smith, Tetragonal bismuth bilayer: a stable and robust quantum spin hall insulator, 2D Mater., 2015, 2, 045010.

70 D. Sánchez-Portal, E. Artacho, J. M. Soler, A. Rubio and P. Ordejón, $A b$ initio structural, elastic, and vibrational properties of carbon nanotubes, Phys. Rev. B: Condens. Matter Mater. Phys., 1999, 59, 12678.

71 W. Harrison and S. Ciraci, Bond-orbital model II, Phys. Rev. B: Solid State, 1974, 10, 1516.

72 S. Cahangirov, M. Audiffred, P. Taug, A. Lacomino, W. Duan, G. Merino and A. Rubio, Electronic structure of silicene on Ag(111): strong hybridization effects, Phys. Rev. B: Condens. Matter Mater. Phys., 2013, 88, 035432. 
73 See ESI†.

74 W. Yu, C. Y. Niu, Z. Zhu, X. Wang and W. B. Zhang, Atomically thin binary $\mathrm{V}-\mathrm{V}$ compound semiconductor: a firstprinciples study, J. Mater. Chem. C, 2016, 4, 6581.

75 M. Y. Liu, Y. Huang, Q. Y. Chen, C. Cao and Y. He, Unexpected electronic structure of the alloyed and doped arsenene sheets: first-principles calculations, Sci. Rep., 2016, 6, 29114.

76 S. Singh and A. H. Romero, Giant tunable Rashba spin splitting in a two-dimensional BiSb monolayer and in BiSb/ AlN heterostructures, Phys. Rev. B, 2017, 95, 165444.
77 Z. H. Benam, H. Arkin and E. Aktürk, Point defects in buckled and asymmetric washboard phases of arsenic phosphorus: a first principles study, Comput. Mater. Sci., 2017, 140, 290-298.

78 S.-S. Li, W.-X. Ji, P. Li, S.-J. Hu, L. Cai, C.-W. Zhang and S.-S. Yan, Tunability of the Quantum Spin Hall Effect in Bi(110) Films: Effects of Electric Field and Strain Engineering, ACS Appl. Mater. Interfaces, 2017, 9, 21515.

79 S.-S. Li, W.-X. Ji, P. Li, S.-J. Hu, L. Cai, C.-W. Zhang and S.-S. Yan, Effect of Amidogen Functionalization on Quantum Spin Hall Effect in Bi/Sb(111) Films, ACS Appl. Mater. Interfaces, 2017, 9, 41443. 